\title{
4SCAR-GD2-modified T-lymphocytes
}

National Cancer Institute

\section{Source}

National Cancer Institute. 4SCAR-GD2-modified T-lymphocytes. NCI Thesaurus. Code C128896.

Genetically modified autologous T-lymphocytes transduced with a lentiviral vector encoding a fourth generation specific chimeric antigen receptor (4SCAR) specific for the disialog ang lioside GD2 and which includes the CD3zeta chain and the signaling domains of the co-stimulatory molecules CD28, CD137, and CD27 fused with the suicide gene inducible caspase 9 (iCasp9), with potential immunomodulating and antineoplastic activities. Upon intravenous administration of 4SCAR-GD2 T-cells, these cells target the GD2 antigen on tumor cells to induce selective toxicity against GD2-expressing tumor cells. The tumor-associated antigen (TAA) GD2 is overexpressed on the surface of neuroblastoma cells and by other neuroectoderm-derived neoplasms, while it is minimally expressed on normal cells. iCasp9 consists of a human FK506 drug-binding domain with an F36V mutation (FKBP12-F36V) linked to human caspase 9. If the administered T-cells lead to unacceptable side effects, the chemical homodimerizer AP1903 can be administered; this binds to the drug binding FKBP12-F36V domain and activates caspase 9, which results in the apoptosis of the administered T-cells and enhances safety of this agent. CD28, CD137 and CD27, T-cell surface-associated co-stimulatory molecules, are required for full $\mathrm{T}$-cell activation. 